\title{
Real-time continuous glucose monitoring in children with critical illness - do we need it?
}

\begin{abstract}
Ahmed El-Nawawy
Despite the tremendous improvement in the management of severe sepsis and septic shock, which are still major problems affecting millions of people around the world each year, mortality is one in four (and often more) and still increasing in incidence..$^{[1]}$ One of the unsolved problems until now is "should we use the tight glycemic control in pediatric sepsis and septic shock?" If the answer is yes then the second question would be "how to monitor blood glucose (BG)?" and this ultimately leads to the third question "what is the consensus about the protocol of insulin administration in this context?"
\end{abstract}

In the recent "International guidelines for management of severe sepsis and septic shock: Surviving sepsis campaign" ${ }^{2]}$ published in 2013, the recommendations for adults differ slightly from those in the pediatric consideration chapter. The recommendation suggests that hyperglycemia in septic shock and severe sepsis should be controlled using a target of $\leq 180 \mathrm{mg} / \mathrm{dl}$ and glucose infusion should accompany insulin therapy in newborns and children. The difference is that the level of recommendation in adults is $1 \mathrm{~A}$ while in pediatrics it is graded $2 \mathrm{C}$. This implies that the BG level should be kept at $180 \mathrm{mg} / \mathrm{dl}$. Infants and children are at higher risk for developing hypoglycemia and a glucose intake of $4-6 \mathrm{mg} / \mathrm{kg} / \mathrm{min}$ or maintenance fluid with dextrose $10 \%$ normal saline containing solution is advised. Children lack glycogen stores, having more insulin resistance and more patients secreting no insulin in shock situation. ${ }^{[3,4]}$ The different American Associations: Clinical endocrinologists, American Heart Association, American College of Physicians and Society of Critical Care Medicine, have published different consensus targeting glycemic

\section{From:}

Department of Pediatrics, Faculty of Medicine, Alexandria University,

Alexandria, Egypt

\section{Correspondence:}

Dr. Ahmed El-Nawawy, Professor of Pediatrics and Pediatric Critical Care, Alexandria University, Alexandria, Egypt.

E-mail: dr_anawawy@yahoo.com

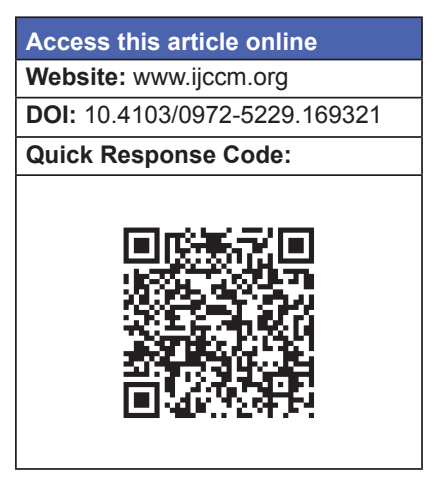

control between 140 and $180 \mathrm{mg} / \mathrm{dl}$. Strangely enough, a survey of pediatric intensivists practice of "Clinical Equipoise Regarding Glycemic Control" (in 2013) of North American Pediatric Intensive Care Unit (PICUs), revealed that the published evidence does not adequately address PICU clinicians concerns.

Pediatric intensivists mostly depend on adult studies because of the small number of randomized controlled trials in pediatrics. The most famous adult study is the "Normoglycemia in Intensive Care Evaluation-Survival using Glucose Algorithm Regulation" study that revealed a slight increase in harm with hyperglycemia control, after a wide meta-analysis. Furthermore, $78 \%$ of intensivists stressed on the need for a multicenter clinical trial for BG control in pediatrics. ${ }^{[5]}$

There are three different methods used in PICU for evaluating BG: Point of care capillary blood testing, venous sampling, and real-time continuous glucose monitoring system (RT-CGMS). The authors, in this study, ${ }^{[6]}$ published

This is an open access article distributed under the terms of the Creative Commons Attribution-NonCommercial-ShareAlike 3.0 License, which allows others to remix, tweak, and build upon the work non-commercially, as long as the author is credited and the new creations are licensed under the identical terms.

How to cite this article: El-Nawawy A. Real-time continuous glucose monitoring in children with critical illness - do we need it?. Indian J Crit Care Med 2015;19:631-2. 
a good comparison between RT-CGMS and venous sampling BG results (as a standard method) among children with septic shock, using percutaneously inserted sensor it evaluates glucose in the interstitial fluid. There has been much criticism for the capillary method and the RT-CGMS. The internationally accepted guidelines of the Surviving Sepsis Campaign ${ }^{[2]}$ explained that several factors affect the accuracy and reproducibility of point of care capillary BG, including type and model of the device used, user expertise, and patient factors: As hematocrit (false elevation in anemia), hypoxia and drugs used as catecholamines, and false elevations over the range, especially hyper and hypoglycemia range. A lot of researchers used RT-CGMS in the design of their studies. ${ }^{[7-11]}$

The author found that the RT-CGMS relative absolute difference (RAD) compare to venous samples results was $17 \%$, another study revealed it to be $13.5 \%$ and it revealed also that RT-CGMS results meeting the "International Organization Standardization" (ISO) were $68.1 \%$ with a better accuracy in septic shock compared to severe sepsis status. ${ }^{[9]}$ The author did not discuss the ISO standards.

Another study compared all three methods for BG evaluation: RT-CGMS values compared to blood gas/glucose analyzer values every $12 \mathrm{~h}$ and to venous BG measurement by central laboratory device as a measured reference. The RAD, strangely enough, was worse in RT-CGMS (14.4\%) compared to blood gas/glucose analyzer $(6.6 \%)$. Contrary to what found in this study, the percentage of matched points in the Clarke error grid zone A was $78.4 \%$ in RT-CGMS, and $98.4 \%$ in blood gas/glucose analyzer, ${ }^{[10]}$ while in this study RT-CGMS matched $94.5 \%$ of readings.

The previous study ${ }^{[11]}$ discussed another missed point in the present study concerning the calibration of the sensor of RT-CGMS. The study revealed that the RAD found within 6 h of sensor calibration was $8.8 \%$, while between 6 and $12 \mathrm{~h}$ after calibration it was significantly high $(20.1 \%)$. This also appeared in the matched points in Clarke error grid zone A to be $92.4 \%$ versus $57.5 \%$. This indicates that RT-CGMS sensor should be calibrated $<6 \mathrm{~h}$, no matter what time interval is recommended by the manufacturer.

The results obtained by RT-CGMS, in the majority of the studies, is affected by situations such as edema (the percutaneous probe is used), shock (skin perfusion affected), large base deficit, therapeutic cooling as well as drug use. ${ }^{[8-10]}$ This point is contrary to what stated by the author in this study.

The last observation is that this study agreed with other studies that RT-CGMS results are not accurate as regards the BG in extremes of hyper and hypoglycemia. Finally, the routine use of RT-CGMS monitoring in ICU is not yet recommended until sufficient studies on the reliability of the system are available. The most important unanswered question in this context is "Do we really need tight BG control that warrants RT-CGMS in severe sepsis and septic shock in PICU?" While certainly possible, one can conclude that the final chapter in this story has not been written.

\section{References}

1. Dombrovskiy VY, Martin AA, Sunderram J, Paz HL. Rapid increase in hospitalization and mortality rates for severe sepsis in the United States: A trend analysis from 1993 to 2003. Crit Care Med 2007;35:1244-50.

2. Dellinger RP, Levy MM, Rhodes A, Annane D, Gerlach H, Opal SM, et al. Surviving sepsis campaign: International guidelines for management of severe sepsis and septic shock, 2012. Intensive Care Med 2013;39:165-228.

3. Garcia Branco R, Tasker RC, Ramos Garcia PC, Piva JP, Dias Xavier L. Glycemic control and insulin therapy in sepsis and critical illness. J Pediatr (Rio J) 2007;83 5 Suppl:S128-36.

4. Vlasselaers D, Milants I, Desmet L, Wouters PJ, Vanhorebeek I, van den Heuvel I, et al. Intensive insulin therapy for patients in paediatric intensive care: A prospective, randomised controlled study. Lancet 2009;373:547-56.

5. Hirshberg EL, Sward KA, Faustino EV, Nadkarni VM, Agus MS, Morris AH, et al. Clinical equipoise regarding glycemic control: A survey of pediatric intensivist perceptions. Pediatr Crit Care Med 2013;14:123-9.

6. Prabhudesai S, Kanjani A, Bhagat I, Ravikumar KG, Bala Ramachandran B. Accuracy of a real-time continuous glucose, monitoring system in children with septic shock: A pilot study. Ind. J Crit Care Med 2015;19:642-7.

7. Fisher JG, Sparks EA, Khan FA, Alexander JL, Asaro LA, Wypij D, et al. Tight glycemic control with insulin does not affect skeletal muscle degradation during the early postoperative period following pediatric cardiac surgery. Pediatr Crit Care Med 2015;16:515-21.

8. Branco RG, Chavan A, Tasker RC. Pilot evaluation of continuous subcutaneous glucose monitoring in children with multiple organ dysfunction syndrome. Pediatr Crit Care Med 2010;11:415-9.

9. Lorencio C, Leal Y, Bonet A, Bondia J, Palerm CC, Tache A, et al. Real-time continuous glucose monitoring in an Intensive Care Unit: Better accuracy in patients with septic shock. Diabetes Technol Ther 2012;14:568-75.

10. Maries G, Konez L, Körner A, Mikos B, Tóth-Heyn P. Role of continuous subcutaneous glucose monitoring in intensive care. Orv Hetil 2013;154:1043-8.

11. Yue XY, Zheng Y, Cai YH, Yin NN, Zhou JX. Real-time continuous glucose monitoring shows high accuracy within 6 hours after sensor calibration: A prospective study. PLoS One 2013;8:e60070. 